\title{
Identifying ESC Failures in Polymers using Fractography
}

\author{
Dale B. Edwards, P.E. ${ }^{1}$ \\ ${ }^{1 .}$ Engineering Systems Inc. (ESI), Aurora, IL, USA
}

It is sometimes very difficult to differentiate between slow crack growth (SCG)/creep rupture and environmental stress cracking (ESC) when observing a fracture surface. There is a wide spectrum of chemical effects on crack growth with different critical stresses for different polymer/chemical combinations. If the critical stress is high, one observes more micro-ductility on the surface. If the critical stress is low, one observes a more glassy fracture surface. Thus, the level of ESC contribution to the failure ranges from very little effect to very aggressive effects. This uncertainty is often reflected in the published resistance tables, as evidenced by the use of terms like "minor effect", "slight corrosion", "moderate effect", "softening", "caution", or a number rating from 1 to 10 for resistance to chemical attack (with 1 representing very poor resistance and 10 representing no effect). [1]

ESC failures resulting from aggressive ESC agents are easy to identify. They have glassy, extremely smooth fracture surfaces and a very low critical stress for the chemical/material combination, as shown in Figure 1, a fracture surface from a CPVC tank exposed to a plasticizer and an ABS part that failed due to exposure to silicone oil. Cracks driven predominantly by stress are also fairly easy to identify with significant micro-ductility on the fracture surface and/or the presence of discontinuous crack growth bands. Figure $2 \mathrm{a}$ is an example of a crack in a CPVC pipe that appears to be more stress-driven, exhibiting micro-ductility on the surface. Figure $2 \mathrm{~b}$ is an example of a mechanically driven crack that occurred due to freezing of water in an assembly of CPVC pipe in the laboratory. This section of pipe was removed and then re-pressurized at high stress (4200 psi) for 1 week before removing it and opening up the fracture surface. This additional time under high stress (4200 psi) caused the rib marking on the fracture surface. The crack had occurred in the piping adjacent to the burst area of the pipe. [2]

An important question is: What level of ESC activity has affected the particular failure under investigation? It can be difficult to discern, based solely on an examination of the fracture surface. Part of the difficulty in distinguishing between SCG/creep and ESC failures hinges upon the definition of ESC. One definition states that a stress crack agent is a chemical that simply enhances creep of the material, enabling the molecules (particularly in the amorphous regions) to slide past one another. The ESC agent lowers the intermolecular forces that help bind the polymer together. The ESC agent does not degrade the material or cause any change in its structure (e.g. molecular weight) and is at best a very weak solvent for the material. This same fracture process happens in creep and creep rupture in the absence of ESC agents. For this reason, some view creep as a special case of ESC in air [3]. The main difference lies in longer times to failure and higher stresses needed to cause failure in SCG/creep rupture versus ESC.

The level of roughness and micro-ductility on the fracture surface is an important indicator of the extent of the role of ESC in the failure. However, the level of micro-ductility present on fracture surfaces due to ESC is different for different polymers. For this reason, although the fracture surface can be very helpful in determining the cause of failure, it does not always give a definitive answer. Rather, other data and testing are often necessary to properly interpret the fracture, and this is especially true for many ESC-related failures. For example, in the event that an ESC agent is present and suspected as a cause or 
contributing factor to the failure, the critical stress for that particular chemical/material combination may provide a clue. If the critical stress is very high, one might simply view these failures as slow crack growth (creep) failures with little or no contribution from the ESC agent. The level of stress acting at the time of failure affects the appearance of the fracture surface and this is true for non-ESC failures, as well. Knowledge of the material's response to both stress and the presence of various environments can be very helpful in determining whether the failure was driven mainly by high stress or by exposure to an ESC agent or another environmental factor. The presence of stress whitening on the fracture surface is an excellent indicator that the yield stress of the material has been exceeded. However, some materials, such as polysulfone, do not exhibit stress whitening upon yielding. As with most aspects of failure analysis, a thorough knowledge of the particular polymer and its failure mechanisms is important in arriving at the root cause of the failure. Fractography is an indispensible tool for failure analysis, but interpretation of fractographic features must be harmonized with known facts about the failure.

\section{References:}

[1] Anon, 1994. Chemical Resistance: Volume I -- Thermoplastics 2nd ed., New York: Plastics Design Library.

[2] Hayes, Edwards, Shah, Fractography in Failure Analysis of Polymers, Elsevier Inc. Oxford, UK, 2015.

[3] D.C. Wright, Environmental Stress Cracking of Plastics, RAPRA Technology, Ltd., Shawbury UK, 1996.

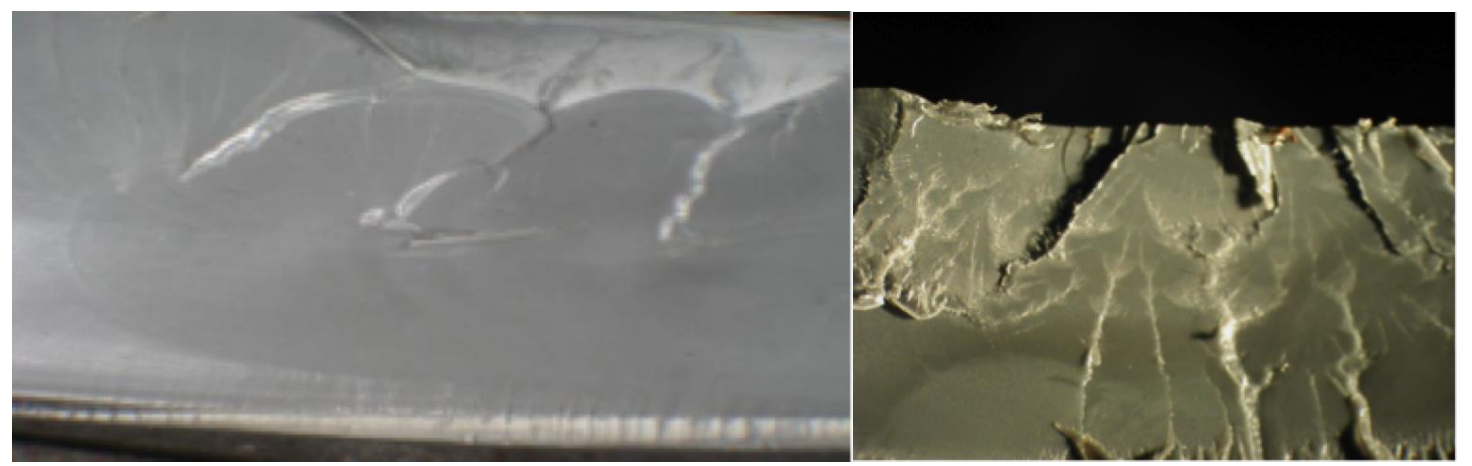

Figure 1. Failures due to an aggressive ESC agent for CPVC (left) and ABS (right).

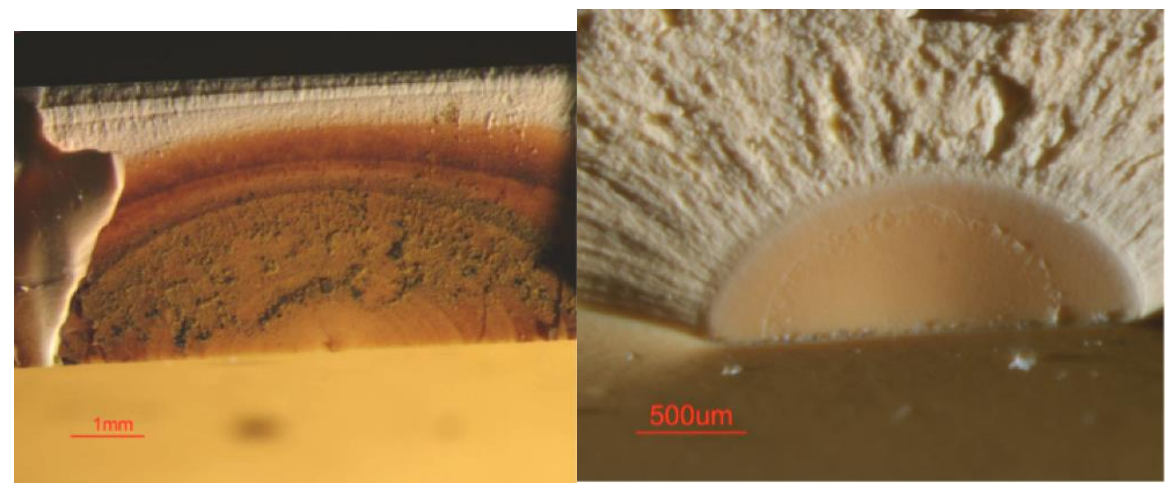

Figure 2a. CPVC failure due to slow crack growth and/or less aggressive ESC agent (left photograph). 2b. Mechanically driven freeze crack from laboratory showing rib marking from secondary loading after freeze event (right photograph). 